\title{
Kinomics Screening Identifies Aberrant Phosphorylation of CDC25C in FLT3-ITD-positive AML
}

\author{
FLORIAN PERNER ${ }^{1,2,3}$, TINA M. SCHNÖDER ${ }^{2,3}$, THOMAS FISCHER ${ }^{1,4}$ and FLORIAN H. HEIDEL FL $^{2,3,4}$ \\ ${ }^{I}$ Department of Hematology and Oncology, Center of Internal Medicine, \\ Otto-von-Guericke University Medical Center, Magdeburg, Germany; \\ ${ }^{2}$ Internal medicine II, Hematology and Oncology, Friedrich-Schiller-University Medical Center, Jena, Germany; \\ ${ }^{3}$ Leibniz-Institute on Aging, Fritz-Lipmann-Institute, Jena, Germany; \\ ${ }^{4}$ Collaborative Research Cluster 854, Medical Faculty, \\ Otto-von-Guericke University Medical Center, Magdeburg, Germany
}

\begin{abstract}
Background/Aim: The presence of FLT3-Internal tandem duplications (ITDs) in human acute myeloid leukemia $(A M L)$ is associated with a dismal prognosis. Altered cell-cycle activity has been reported in FLT3-ITD-positive AML; however, the mechanisms by which this oncogene influences cell-cycle activity remained so far elusive. Materials and Methods: A phospho-kinomic screen was used to identify downstream effectors of FLT3-ITD. Validation and functional characterization was performed by western blotting, cell-cycle analysis and apoptosis assays. Results: We identified aberrant phosphorylation of CDC25C-T48 in FLT3-ITD mutated cells. Forced expression of CDC25C affected cell-cycle progression but did not affect sensitivity to cellular stress. Conclusion: Depending on the oncogenic background, CDC25C may reveal protective or oncogenic functions. Our results identify CDC25C as a downstream target of the mutated tyrosine kinase FLT3ITD affecting cell-cycle regulation in a model of AML.
\end{abstract}

Acute myeloid leukemia (AML) is a genetically heterogeneous disease characterized by clonal selection and accumulation of mutations during disease progression (1). FLT3 is one of the most frequently mutated genes in adult AML (2-4) that encodes for a receptor tyrosine kinase required for maintenance of normal hematopoiesis (5). During leukemia development, the occurrence of FLT3-internal tandem duplications (ITD) induces constitutive activation of the encoded receptor tyrosine kinase

This article is freely accessible online.

Correspondence to: Professor Dr. Florian H. Heidel, Innere Medizin II, Hämatologie und Onkologie, Universitätsklinikum Jena, Am Klinikum 1, 07747 Jena, Germany. Tel: +49 3649324673, Fax: +49 36419324212, e-mail: florian.h.heidel@gmail.com

Key Words: FLT3-ITD, CDC25C, Ser216, Thr48, AML, cell cycle. and leads to activation of downstream effectors, such as STAT5, ERK and AKT. Depending on cooperating oncogenic events, presence of FLT3-ITD may be associated with a poor prognosis in patients with $\operatorname{AML}(2,6,7)$. Recent reports also suggested that constitutive activation of FLT3-signaling might result in aberrant cell-cycle activity (8).

Stringent regulation of cell-cycle activity is of major importance to ensure proper development of hematopoiesis and to orchestrate tissue regeneration. Conversely, deregulation of cell cycle-relevant proteins can promote the development of cancer $(9,10)$. The final steps during cell-cycle transition are controlled by cyclin-dependent kinases (CDKs) in complex with the appropriate cyclins. CDKs are preserved in an inactive state through phosphorylation of distinct amino acid residues. Several amino acid residues need to be dephosphorylated by cell division cycle 25 (CDC25) phosphatases $(9,10)$ to activate CDKs. Therefore, CDC25 phosphatases are essential regulators of cell cycle progression in malignant and non-malignant cells. In mammals, this group of phosphatases consists of three homologues, CDC25A, CDC25B and CDC25C. These are involved in pathogenesis of human malignancies $(9,10)$ and, specifically, myeloid leukemia $(11,12)$. While CDC25A is important for S-phase entry and, crucial for regulating cellular proliferation (13), $\mathrm{CDC} 25 \mathrm{~B}$ and $\mathrm{CDC} 25 \mathrm{C}$ are believed to control mitosis entry (14). Taken together, all CDC25 phosphatases are involved in cell-cycle checkpoint regulation with redundant and non-redundant functions (9). Recently, frequent mutation of $\mathrm{CDC} 25 \mathrm{C}$ has been reported in patients with familial platelet disorder (FPD). CDC25C defines a preleukemic clone, which eventually acquires additional mutations during disease progression. Of note, the observed CDC25C mutations are exclusively found in FPD patients rather than in de novo AML or myelodysplastic syndromes (MDSs) (15). Here, we provide first evidence that FLT3-ITD induces aberrant phosphorylation of $\mathrm{CDC} 25 \mathrm{C}$ that functionally mimics features observed in the mutant $\mathrm{CDC} 25 \mathrm{C}$ isoforms of FPD patients. 


\section{Materials and Methods}

Cell lines and cell culture. The human AML cell line Molm13 and murine 32D cells were cultured in RPMI1640 medium (PAA, Cölbe, Germany) supplemented with $10 \%$ FBS (PAA) in a humid atmosphere of $5 \% \mathrm{CO}_{2}$ at $37^{\circ} \mathrm{C}$.

Kinase profiling. For kinase profiling we used an established cell line model (32D) harboring an FLT3-ITD mutation that enables cytokine-independent growth (16). 32D-FLT3-ITD cells were cultured for 24 hours in RPMI under steady-state conditions prior to FLT3-tyrosine-kinase-inhibitor (TKI) PKC412 (midostaurin) exposure (or to DMSO as diluent control). One complication of pharmacologic FLT3-inhbition experiments in 32D-FLT3-ITD cells is requirement of FLT3-signaling for cell survival. Accordingly, we incubated 32D cells with midostaurin or diluent control 'shortterm' for 30 minutes, which is sufficient for abrogation of FLT3mediated signaling but not sufficient for induction of apoptosis. Following drug exposure, cells were lysed and labeled with ${ }^{33} \mathrm{P}$-ATP. Lysates were hybridized on the Pepscan 'kinomics slide' for 2 hours at $37^{\circ} \mathrm{C}$ in a humid chamber. Binding of active kinases to the corresponding peptide sequences of downstream effectors results in incorporation of radioactively labeled ${ }^{33} \mathrm{P}-\mathrm{ATP}$ as a substrate. Radioactively labeled ${ }^{33} \mathrm{P}-\mathrm{ATP}$, thereby, enables detection of kinase activity. Following several washing steps, the slide was exposed for 7 days to a Pospho-Storage-Screen ${ }^{\mathrm{TM}}$ (GE Healthcare, Chalfont St Giles, UK). Analysis was performed on a Typhoon Trio Plus Imager ${ }^{\mathrm{TM}}$ (GE Healthcare). Phosphopeptide arrays may be limited by unspecific binding of kinases to their substrates; however, several bona fide substrates/peptides for FLT3-kinase are available on these slides and can be used for validation. Positive (scrambled substrates) and negative controls (mixed peptides without serine, threonine or tyrosine residues) were used for calculation of background noise or insufficient binding using the PepChip Kinomics grid (www.pepscan.com).

Western blotting. For Western blotting analysis we used whole cellular lysates (17), as well as nuclear extracts that were harvested using the ProteoExtract ${ }^{\circledR}$ kit (Merck Chemicals, Darmstadt, Germany) according to the manufacturer's instructions.

Retroviral transduction. Retroviral transduction of 32D cells was performed as described previously (15). For the production of retroviral particles, HEK293T cells were cultured to approximately $70 \%$ confluence. Retroviral infection was performed as previously described (17). In brief, $1 \times 10^{6} 32 \mathrm{D}$ cells were washed twice in PBS before being re-suspended with $1 \mathrm{ml}$ of virus containing medium in a 50-ml tube. The cells were centrifuged for $90 \mathrm{~min}$ at $33^{\circ} \mathrm{C}$ at $2,000 \mathrm{rpm}$ and left with the virus in culture overnight in a $24-w e l l$ plate. The next day, the cells were washed and transferred to RPMI culture medium.

Cell cycle and apoptosis. Cell cycle analysis in 32D cells was performed by flow cytometry using a co-staining with Ki67 (phycoerythrin/cyanine7 (PE/Cy7); Biolegend, London, UK) and Hoechst 33342 as previously described (17). For the detection of apoptosis we used a co-staining of AnnexinV (allophycocyanin (APC)-labeled antibody; Biolegend) and SytoxBlue ${ }^{\circledR}$ dead cell stain (Life technologies, Darmstadt, Germany) in flow cytometry. The measurements were performed using a FACS Canto II cytometer and FACS Diva software (BD Bioscience, Heidelberg, Germany). Data analysis was conducted using Flow Jo ${ }^{\circledR}$ software (TreeStar, Ashland, OR, USA).

Statistical analysis. The generation of graphs and statistical analysis was performed using GraphPad Prism Software (GraphPad Software Inc, La Jolla, CA, USA).

\section{Results}

CDC25C is aberrantly phosphorylated in FLT3-ITD-positive cells. FLT3 length mutations (ITD's) phosphorylate and activate major signaling pathways, such as STAT5, ERK and AKT. To gain an unbiased view on signaling events that may influence cell cycle control, we used a semi-quantitative phospho-kinomic screen to explore FLT3-ITD-controlled protein phosphorylation pathways (Figure 1A). Peptide kinome array technology is a method that currently allows quantification of kinase activity in a large subset of kinases on commercially available chip platforms. Each array displays as many as 1,152 individual peptide kinase substrates in triplicate on a glass slide and allows investigation of kinase substrate preference, identification of substrates, with best selectivity against a kinase panel, and is suitable for characterization of kinase function. The substrates are all stress-, growth- or differentiation-related. When applying lysates from 32D-FLT3-ITD cells with or without pharmacologic inhibition of FLT3 kinase, T48 of CDC25C appeared to be strongly phosphorylated in the untreated cells and was subsequently reduced when treated with the FLT3 inhibitor Midostaurin (Figure 1B). This residue has been described as an important positive regulator of CDC25C activity at mitosis entry (18). Under physiologic conditions, Thr48 phosphorylation is tightly regulated by the cell cycle machinery and exclusively activated before mitosis (18-20). The presence of FLT3-ITD seems to interrupt this regulation leading to aberrant phosphorylation of CDC25CT48 in interphase cells, while inhibition of FLT3-ITD using midostaurin (PKC412) reverses this alteration. We calculated the relative phosphorylation level of the CDC25Cphosphosites after treatment with PKC412 compared to diluent control (Figure 1C). We found significant reduction ( $>50 \%$ of baseline) of T48 phosphorylation $(p=0.0012)$. Of note, serine residue 216 , which has been described as an amino acid residue relevant for cell cycle control in several cellular backgrounds, remained largely unaffected. To validate our findings in human cells, we investigated cell signaling in a human myeloid leukemia cell line (MOLM13).

First, we confirmed inhibition of CDC25C-T48 phosphorylation upon FLT3-inhibitor treatment. Exposure to $50 \mathrm{nM}$ of the selective FLT3-inhibitor quizartinib (AC220) resulted in rapid abrogation of FLT3-, ERK- and CDC25CT48 phosphorylation (Figure 2A, left panel). As ERK signaling influences CDC25 activity in various cell types (18), 


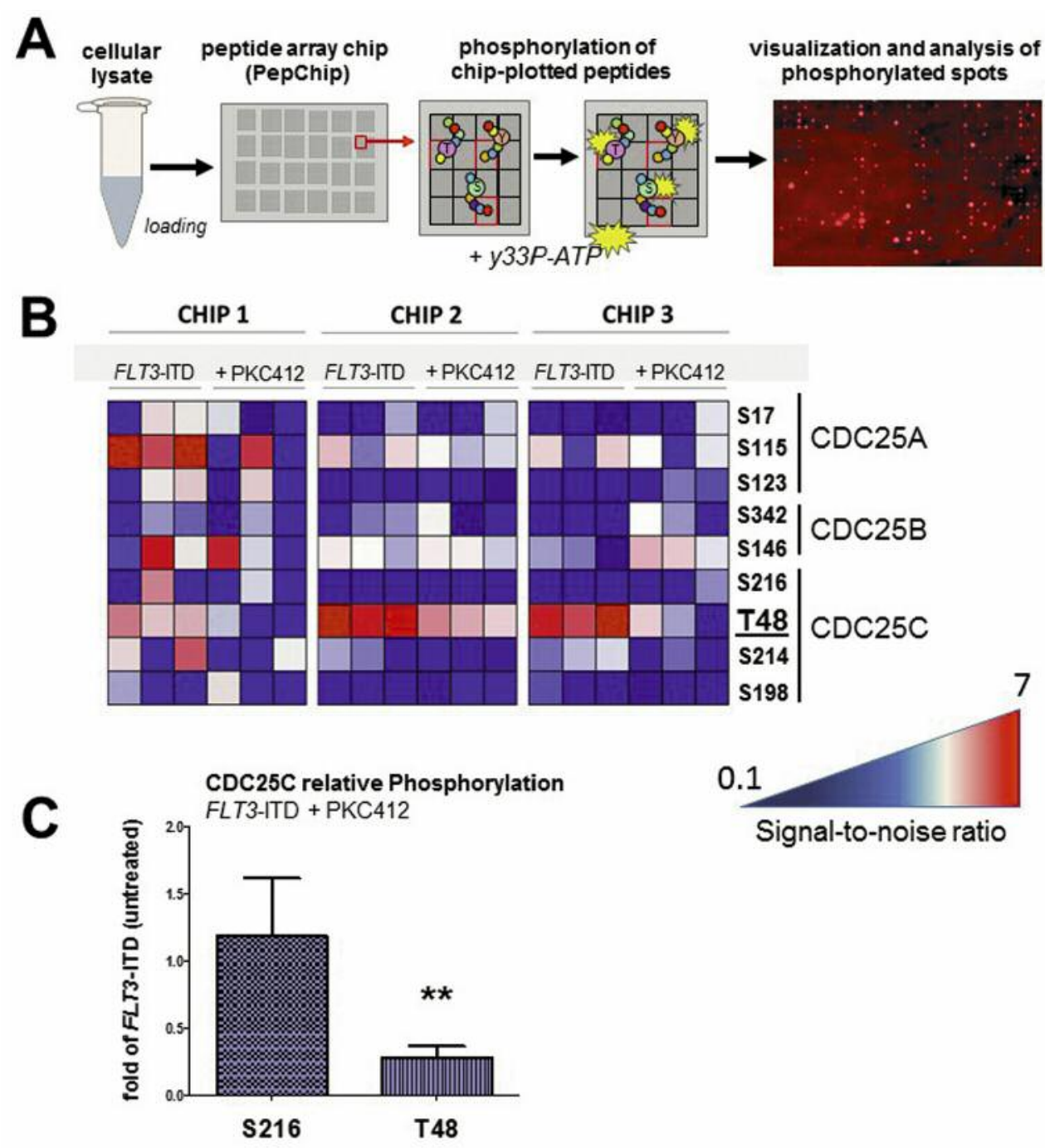

Figure 1. Phospho-kinomic screen identifies CDC25C-T48 as a downstream target of FLT3-ITD. (A) Schematic illustration of the phospho-kinomic screen and data analysis performed on 32D-FLT3-ITD cells. Protein lysates were hybridized on the array. (B) Heat map of CDC25 phosphorylation sites in FLT3-ITD-positive cells (untreated versus PKC412 (100 nM) treated). Signal-to-noise ratios of each chip ( $n=3)$ are depicted. (C) Relative changes in CDC25C phosphorylation following pharmacologic FLT3-inhibition.

we aimed to clarify whether inhibition of MEK1/2 would be sufficient to inhibit phosphorylation of T48. Application of the MEK-inhibitor UO126 was effective and sufficient to reduce ERK and CDC25C-T48 phosphorylation (Figure 2A, right panel). Therefore, we propose that phosphorylation of T48 is mediated by FLT3-ITD signaling and is dependent on downstream MEK/ERK activation. Due to its highlighted role in regulating cellular localization and activation of CDC25C (21-23), we also investigated phosphorylation of CDC25CS216. Phosphorylation of CDC25C-S216 was not significantly altered by short-term exposure to PKC412 (Figure 1B). Consistent with the kinomics data, phosphorylation of S216 was very weak in FLT3-ITD-positive MOLM13 cells (Figure 2B). However, following pharmacologic inhibition of FLT3kinase, phosphorylation of S216 was restored within one hour after treatment. To assess for a potential association of S216 phosphorylation with subcellular localization of CDC25C, we also prepared nuclear lysates (Figure 2C). Following treatment of MOLM13 cells with quizartinib we observed a decrease in nuclear CDC25C at 2 and $4 \mathrm{~h}$ after treatment, which confirms previously published reports (24).

FLT3-ITD signaling impairs cell-cycle control in a two-hit model of $A M L$. To elucidate on the functional consequences of 

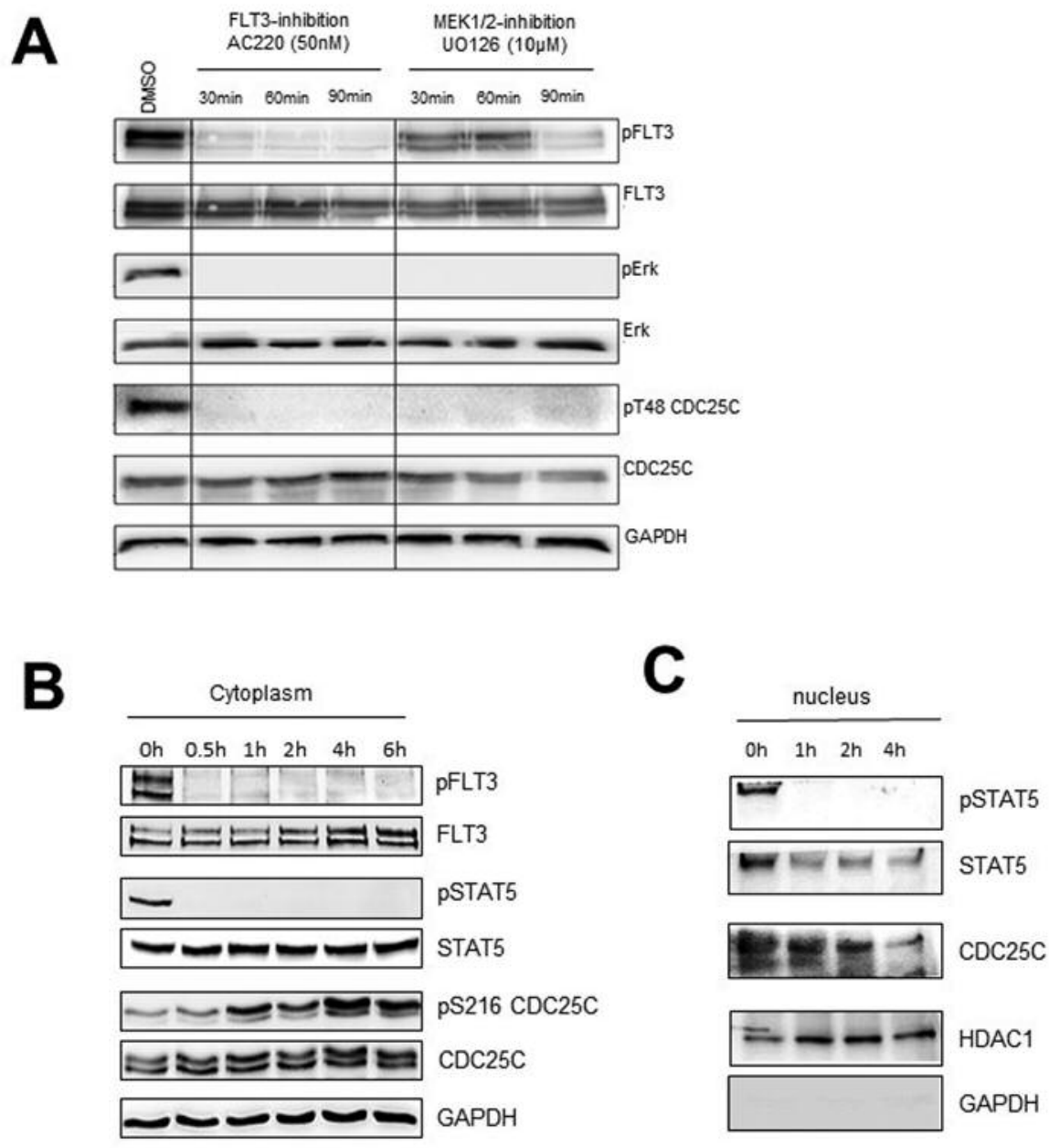

Figure 2. CDC25C phosphorylation and localization is aberrantly regulated by FLT3-ITD. (A) Western blot analysis of protein lysates derived from human AML cell lines (MOLM13, FLT3-ITD-positive) after treatment with DMSO as a soluble control, the FLT3 inhibitor quizartinib (AC220) or the MEK1/2-inhibitor UO126. Cells were incubated with each inhibitor for 30, 60 and 90 minutes before lysis and detection of FLT3, ERK and CDC25C-T48 phosphorylation. (B) Western blot analysis of MOLM13 cell lysates after treatment with 50 nM AC220. (C) Western blot analysis of nuclear protein extracts.

CDC25C expression in FLT3-ITD mutated cells, we generated a cellular model of AML to address genetic heterogeneity and clonal diversity of the disease. We used 32D cells and stably transduced them with FLT3-WT or FLT3-ITD constructs. Subsequently, the MLL-AF9 fusion protein was co-expressed (pMSCV-Neo) to recapitulate a two-hit model (Figure 3A). This oncogenic background is comparable to MOLM13 cells, which are $M L L-A F 9$ - and FLT3-ITD-positive, and cooperation between FLT3-ITD and MLL-AF9 to induce a potent leukemia phenotype has been reported recently (25). Moreover, we transduced the respective cell lines either with an MSCV-based construct expressing CDC25C or empty vector control.

This enabled us to study the influence of forced CDC25C expression on cell cycle regulation in non-oncogenic cells (FLT3-WT) and transformed cells: FLT3-WT+MLL-AF9 or
FLT3-ITD (single hits); and FLT3-ITD+MLL-AF9 (double hit) (Figure $3 \mathrm{~A}$ ). CDC25C expression was controlled by green fluorescent protein (GFP) measurement using flow cytometry (Figure 3B) and by gene expression analysis using quantitative real-time polymerase chain reaction (PCR) (Figure 3C).

We performed cell-cycle analysis by flow cytometry (Ki-67 and Hoechst 33342 co-staining) to identify potential alterations in cell cycle activity under steady-state conditions (Figure 4A). As we used cell lines cultured in vitro, percentage of $\mathrm{G}_{0}$-phase cells appeared rather low (2-3\%) with no major differences between the different genetic backgrounds analyzed (Figure 4B). CDC25C overexpression did not affect cell cycle activity in FLT3-WT cells. In the presence of $M L L$ AF9 ("single hit"), forced CDC25C expression results in a 


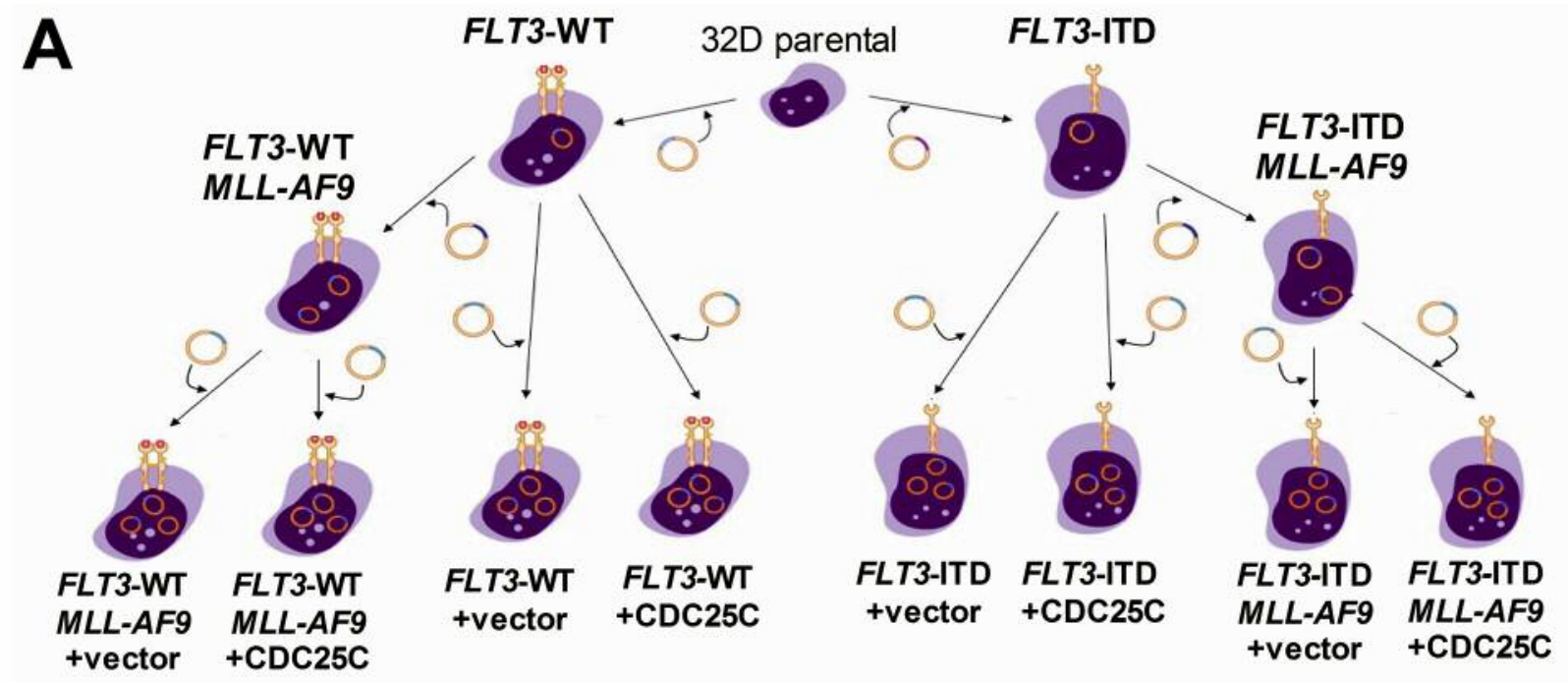

B
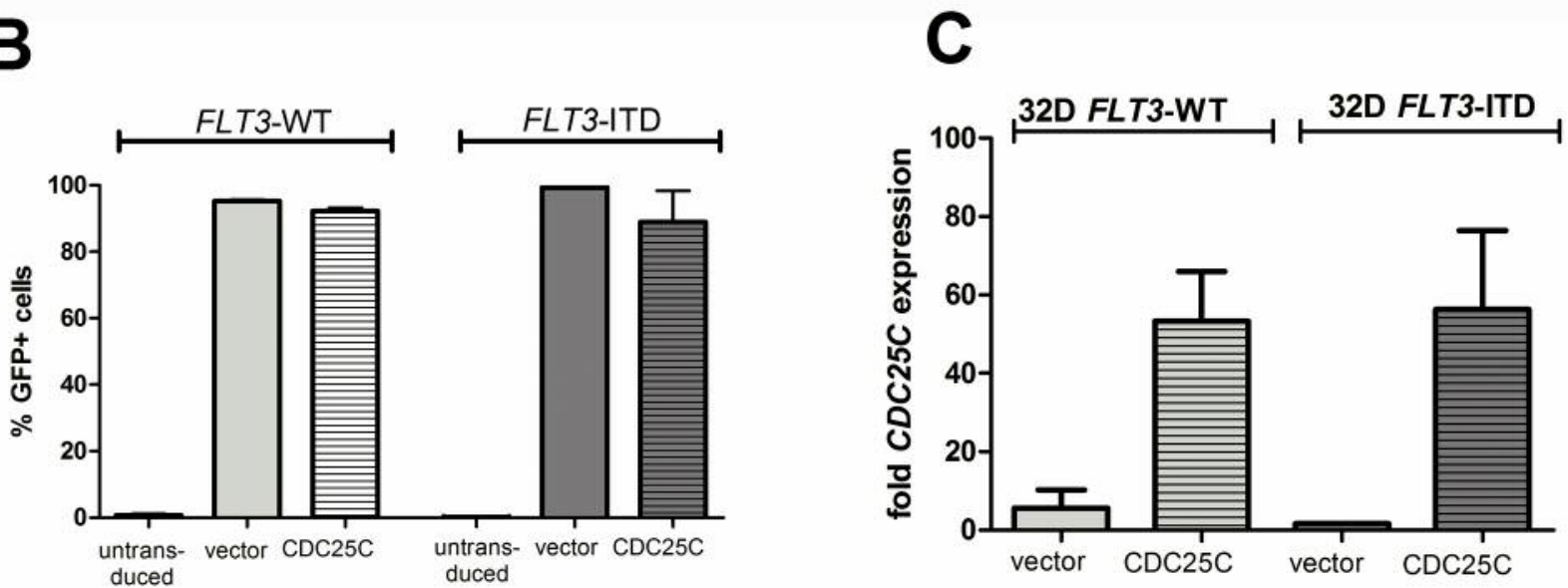

Figure 3. Generation of a cellular model to study CDC25C function downstream of FLT3-ITD. (A) Schematic view demonstrating generation of the two-hit-model of AML in 32D cells. (B) Flow cytometry analysis to control for CDC25C-GFP expression in transduced 32D cells. (C) Quantitative real-time PCR analysis using Taqman ${ }^{\circledR}$ validated primers confirms overexpression of CDC25C in FLT3-WT-and FLT3-ITD-expressing cells.

delay of cell cycle activity and increased abundance of cells in $\mathrm{S}_{-} \mathrm{G}_{2}-\mathrm{M}$ (Figure $\left.4 \mathrm{C}\right) \quad(p=0.0383)$. These cells may eventually undergo $\mathrm{G}_{2}$-arrest due to cellular stress caused by introduction of the $M L L-A F 9$ oncogene. Thus, we found a reduced number of cells in $\mathrm{G}_{1}$ (Figure 4D) $(p=0.05)$. When we introduced FLT3-ITD on top of $M L L-A F 9$ into the cellular system, the effect on cell cycle appears abrogated. Finally, we observed a reduction of cells in $\mathrm{S}_{-} \mathrm{G}_{2}-\mathrm{M}$ phase, even in the presence of $M L L-A F 9$ (Figure $4 \mathrm{C}, \mathrm{D})(p=0.0011)$. Taken together, these findings suggest distinct requirement and influence of $\mathrm{CDC} 25 \mathrm{C}$ depending on the genetic background.

Expression of CDC25C does not influence sensitivity to targeted therapies or cytotoxic drugs. To clarify, whether
CDC25C expression could exert protective effects on cells treated with kinase inhibitors (TKI) or cytotoxic drugs, we studied the induction of apoptosis in the 32D cell model. The percentage of apoptotic cells was determined by AnnexinV/Sytox-Blue co-staining using flow cytometry. First, we analyzed spontaneous apoptosis under steady-state conditions and observed increased levels of steady-state apoptosis in cells with one or more mutations compared to FLT3-WT controls. This may be attributed to an increase in cellular stress due to the increased proliferative capacity or genomic instability. However, no difference was detectable between cells with and without $\mathrm{CDC} 25 \mathrm{C}$ expression independent on the genetic background. (Figure 5A). To investigate, whether CDC25C overexpression could confer 
A
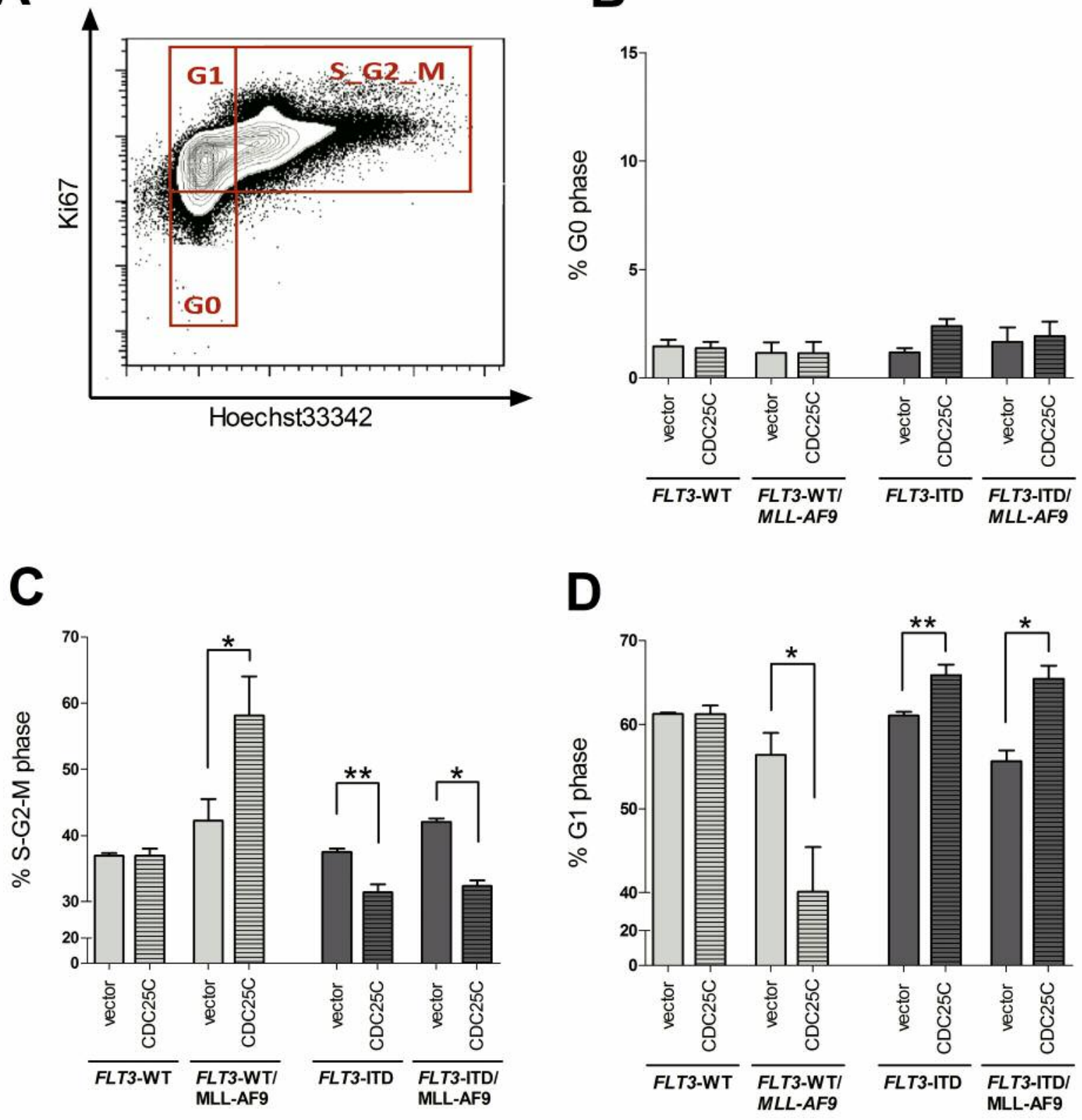

Figure 4. Forced expression of CDC25C influences cell-cycle regulation. (A) Representative gating strategy to assess for cell cycle activity (Ki67/Hoechst33342). Gating and analysis was performed using FlowJo ${ }^{\circledR}$ software. (B, C, D) Distribution of cells in (B) G0, (C) S-G $2^{-M-a n d ~(D) ~}$ $G_{1}$-phase $(n>/=4)$.

stress resistance, we performed interleukin (IL)-3 starvation in 32D FLT3-WT cells transduced with CDC25C (or empty vector control) for $48 \mathrm{~h}$. Overexpression of CDC25C did not confer any protective effect on cell survival in this setting (Figure 5B). To further elucidate on functional consequences of forced CDC25C expression, we applied targeted therapies (FLT3-inhibitor treatment) or cytotoxic drugs (cytarabine, daunorubicine) that are clinically used to treat acute myeloid leukemia. Following treatment with $50 \mathrm{nM}$ of the FLT3Inhibitor AC220, FLT3-ITD-mutated cells underwent apoptosis within $48 \mathrm{~h}$ irrespective of CDC25C expression (Figure 5C). Even at low concentrations of AC220 (5 nM), no difference was detectable in CDC25C-expressing cells compared to empty vector control (Figure 5D). Likewise, upon treatment with cytotoxic agents, such as cytarabine and daunorubicin, the percentage of apoptotic cells remained 


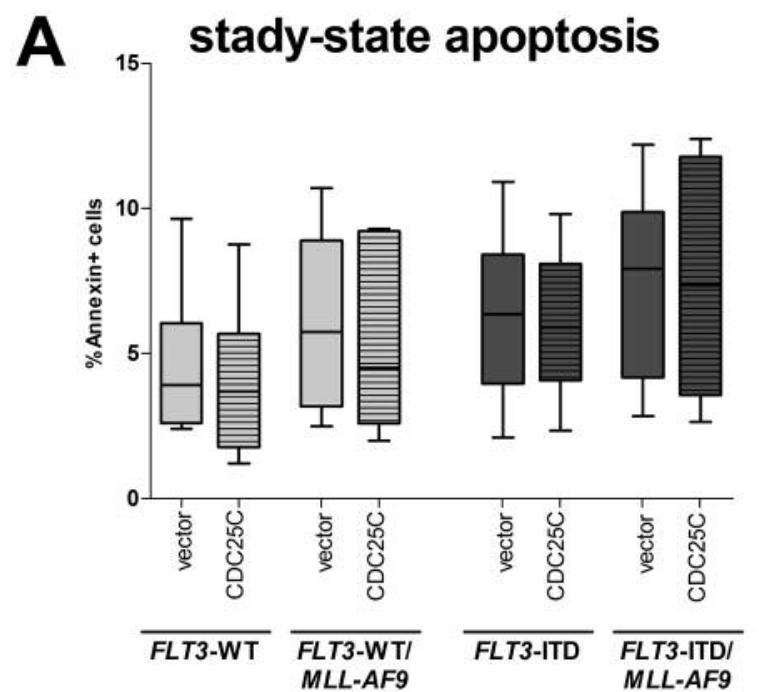

C FLT3-ITD cells: AC220 (50nM)

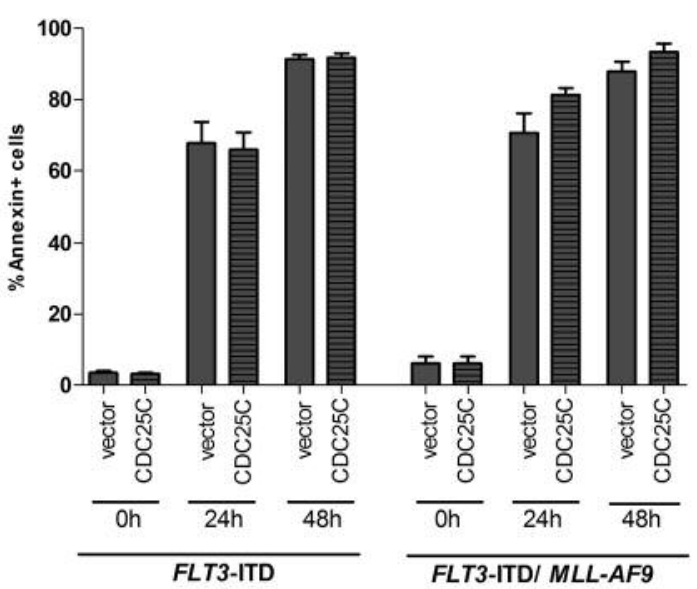

B FLT3-WT cells: IL-3 starvation

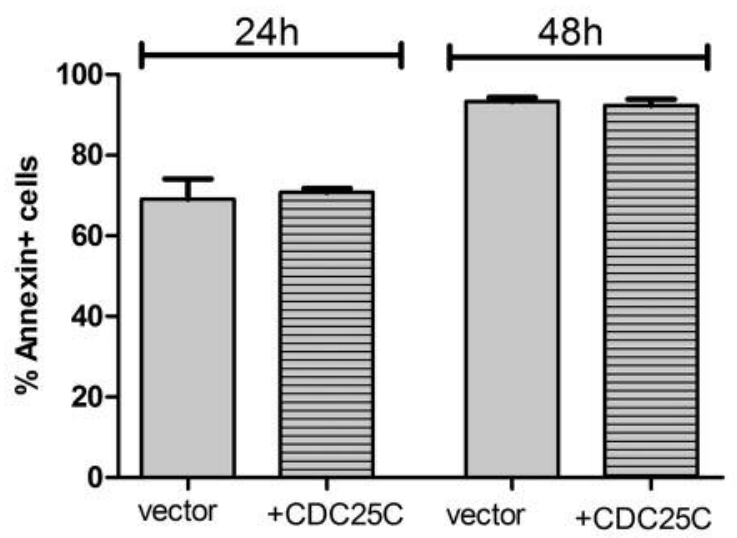

D FLT3-ITD cells: AC220 (5nM)

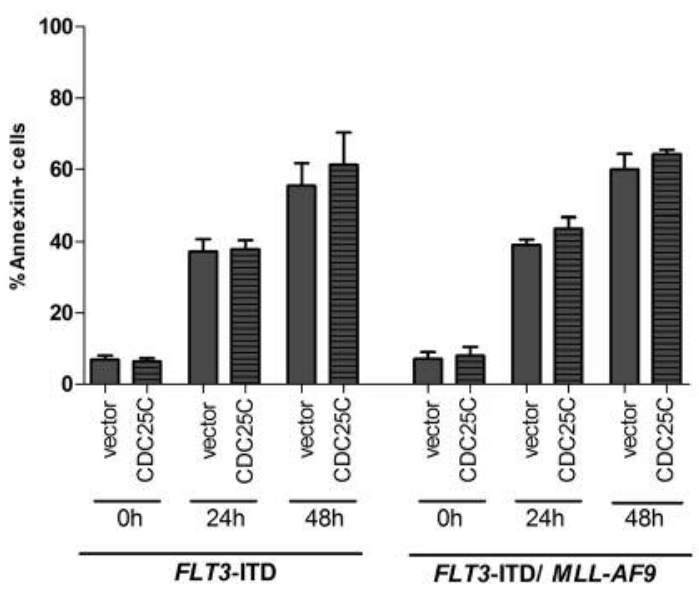

E cytostatic drug treatment - Daunorubicine (DNR), Cytarabin (Ara-C)
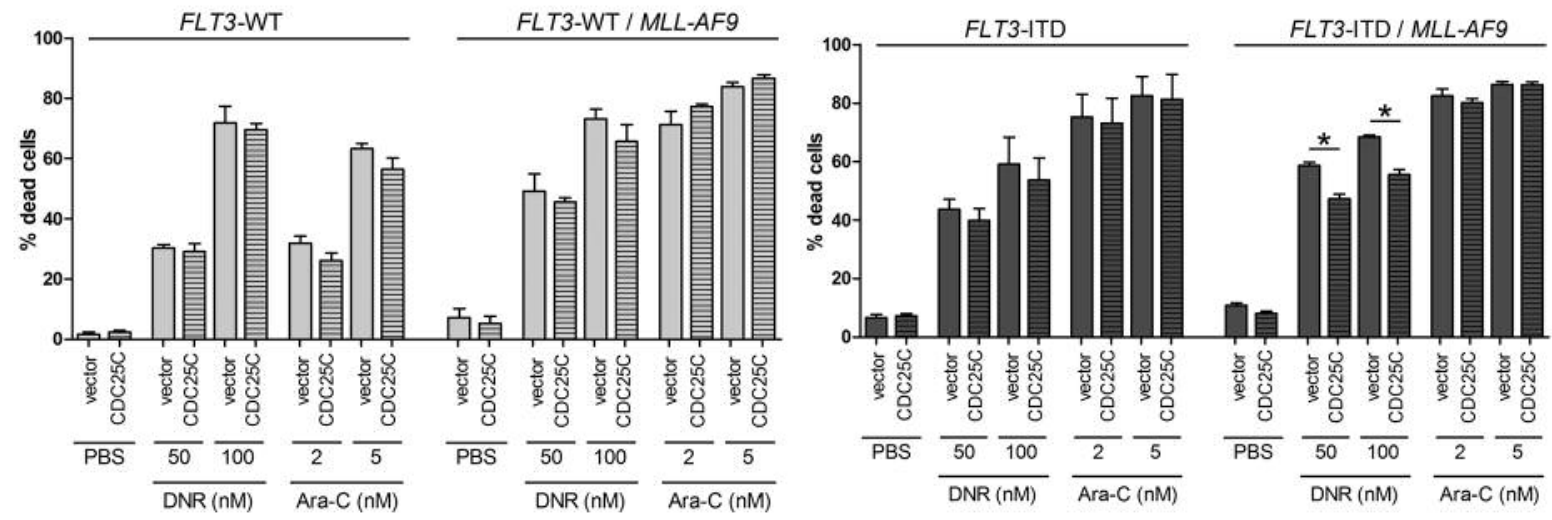

Figure 5. Forced expression of CDC25C does not affect susceptibility to cell stress. (A) Apoptosis of cells as detected by AnnexinV-positive cells by flow cytometry during steady state. (B) Apoptosis in FLT3-WT-expressing 32D cells upon IL-3 starvation. (C, D) Induction of apoptosis in FLT3ITD-positive cell lines following pharmacologic inhibition with $(C)$ high doses $(50 \mathrm{nM})$ or $(D)$ low doses $(5 \mathrm{nM})$ of quizartinib (AC220). (E) Induction of apoptosis in FLT3-WT- (left panel) and FLT3-ITD-(right panel) expressing cells when exposed to cytotoxic drugs. Daunorubicin (DNR) and cytarabine (AraC) were used at the indicated concentrations for $24 \mathrm{~h}$. 


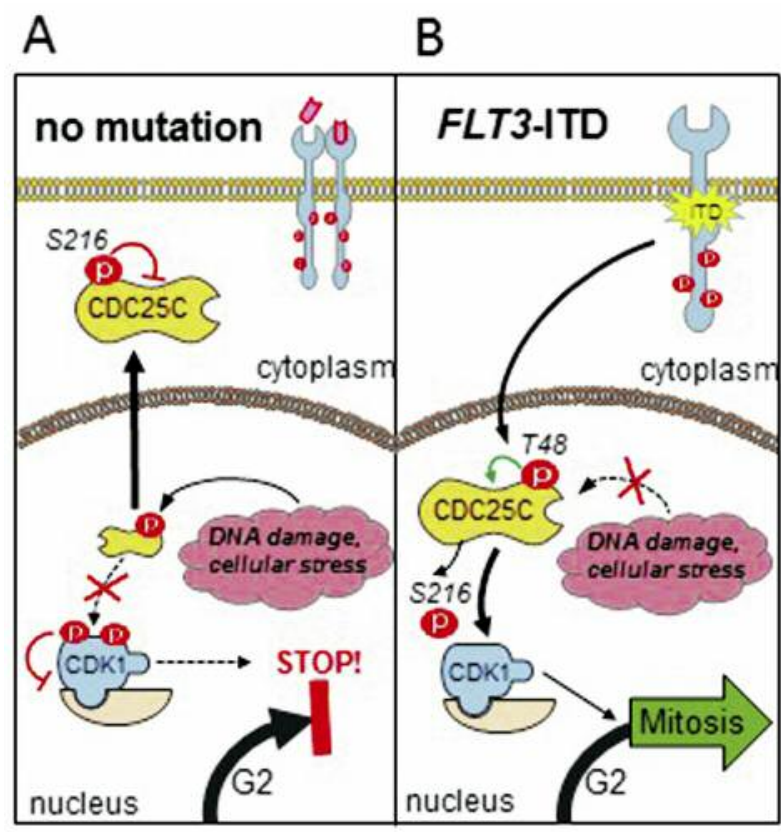

Figure 6. Proposed schematic view of CDC25C function depending on the mutational background. (A) FLT3-wild type conditions: CDC25C function is tightly regulated during cell cycle. In the presence of DNA damage, CDC25C is strictly localized to the cytoplasm by constant Ser216 phosphorylation to prevent premature mitosis entry. (B) FLT3ITD-positive cells: the inhibitory Ser216 residue becomes inactive during interphase, while the activating phospho-site Thr48 is constantly phosphorylated and may result in aberrant activation of CDC25C.

largely unaffected irrespective of CDC25C expression (Figure 5E). Cells harboring a "double hit" (MLL-AF9 and FLT3-ITD) revealed a slight reduction in cell death after daunorubicin treatment when CDC25C was overexpressed (Figure 5E). This rather subtle effect could reflect the increasing importance and impact of cell cycle regulators (such as CDC25C) in cells that acquire several mutational events during clonal selection. Taken together, forced expression of CDC25C did not show any major deleterious or protective effects on cells that were exposed to cytotoxic stress.

\section{Discussion}

Implementing our findings and data from the literature, we propose a model explaining how CDC25C function may be influenced by FLT3-ITD signaling (Figure 6). In nontransformed cells (e.g., 32D-FLT3-WT cells), CDC25C is located in the cytoplasm during interphase and becomes recruited to the nucleus before mitosis to orchestrate cell-cycle progression. Following cytotoxic stress or DNA damage, CDC25C contributes to the protection of genomic integrity in non-mutated cells by strict cytoplasmic localization to prevent mitosis entry (Figure 6A). Constitutive activation of FLT3signaling (e.g., by activating FLT3-ITD mutations) may impair protective functions of $\mathrm{CDC} 25 \mathrm{C}$ through alteration of posttranslational modifications. Thr48 serves as a positive regulator of $\mathrm{CDC} 25 \mathrm{C}$ function and is constantly phosphorylated during interphase depending on activation of ERK activity (downstream of FLT3-ITD). Pharmacologic inhibition of either FLT3 or MEK1/2 results in reduction of CDC25C-T48 phosphorylation. In contrast, amino acid residue Ser216, which is probably the most important negative regulator of $\mathrm{CDC} 25 \mathrm{C}$ activity, remains repressed under the influence of FLT3-ITD and becomes activated following FLT3-kinase inhibition. As a result of this phosphorylation, CDC25C appears to be permanently localized in the nucleus (Figure 6B). Overexpression of CDC25C in FLT3-WT cells did not result in major alteration of cell cycle activity, which may indicate minor functional relevance in non-mutated cells. However, alterations in cell cycle activity became evident upon introduction of different oncogenes: $M L L-A F 9$ and/or FLT3-ITD. While $M L L-A F 9$ led to reduction of cell cycle activity, FLT3-ITD seemed to reverse the $M L L-A F 9$-induced delay (Figure 4C, D). These findings suggest that aberrant phosphorylation of CDC25C caused by FLT3-ITD may become functionally relevant for cell cycle regulation in leukemic cells. Furthermore, the ability of FLT3-ITD to escape from cell cycle arrest under mutational stress could be a mechanism supporting the accumulation of genetic alterations, thereby, promoting disease progression in FLT3ITD mutated AML. Therefore, it is tempting to speculate on FLT3-ITD-induced changes in CDC25C-phosphorylation that mimic those observed for the CDC25C mutants in FPD-AML patients (15). Our observation, that forced expression of CDC25C did neither influence susceptibility to cell stress nor apoptosis, is also consistent with the findings of Yoshimi et al. where $\mathrm{CDC} 25 \mathrm{C}$ mutated clones in the FPD patients disappeared in AML in the presence of more competitive clones, such as GATA2 mutated cells. Impairment of cell cycle control may be caused by aberrant phosphorylation of CDC25C downstream of FLT3-ITD. Aberrations in cell cycle control may promote accumulation of additional mutations and influence clonal progression to AML (26). In patients with MDSs, $C D C 25 C$ is part of the commonly deleted region on chromosome $5 q(27,28)$. Furthermore, alternative splicing of $C D C 25 C$ has been observed in bone marrow samples from patients with MDS (29) and downstream of the SRSF2-P95 mutant (30). It appears that $\mathrm{CDC} 25 \mathrm{C}$ may be recurrently deregulated in MDS patients, indicating a potential involvement in development of pre-leukemic clones. Regulation of CDC25C-T48 phosphorylation was dependent on ERK signaling in our model. As the MEK/ERK pathway is frequently mutated or aberrantly activated in a variety of cancers, CDC25C may also be involved in cell cycle alterations of Ras-mutated solid cancers. Consistent with our 
observations, that $\mathrm{CDC} 25 \mathrm{C}$ overexpression has no impact on WT cells, conventional knockout of $C D C 25 C$ in a mouse model did not reveal any major phenotype (31), probably due to compensatory effects of the other CDC25 family members. The fact that changes in CDC25C expression seem to be of minor importance for normal cells, while having major influence on cancer cells, makes it an interesting signaling node for targeted therapies.

\section{Conflicts of Interest}

The Authors have nothing to disclose.

\section{Acknowledgements}

The Authors thank T.S. Mack for advice during the first steps of this project. This work was supported by grants of the DFG Graduate School GRK1167 to F.P. and T.F. (TP17med) and the DFGCollaborative Research Cluster (CRC854) to T.F. and F.H.H (Project A20).

\section{References}

1 Cancer Genome Atlas Research N: Genomic and epigenomic landscapes of adult de novo acute myeloid leukemia. N Engl J Med 368: 2059-2074, 2013.

2 Frohling S, Schlenk RF, Breitruck J, Benner A, Kreitmeier S, Tobis K, Dohner H, Dohner K and leukemia AMLSGUAm: Prognostic significance of activating FLT3 mutations in younger adults (16 to 60 years) with acute myeloid leukemia and normal cytogenetics: a study of the AML Study Group Ulm. Blood 100: 4372-4380, 2002.

3 Kottaridis PD, Gale RE, Frew ME, Harrison G, Langabeer SE, Belton AA, Walker H, Wheatley K, Bowen DT, Burnett AK, Goldstone AH and Linch DC: The presence of a FLT3 internal tandem duplication in patients with acute myeloid leukemia (AML) adds important prognostic information to cytogenetic risk group and response to the first cycle of chemotherapy: analysis of 854 patients from the United Kingdom Medical Research Council AML 10 and 12 trials. Blood 98: 1752-1759, 2001.

4 Nakao M, Yokota S, Iwai T, Kaneko H, Horiike S, Kashima K, Sonoda $\mathrm{Y}$, Fujimoto $\mathrm{T}$ and Misawa $\mathrm{S}$ : Internal tandem duplication of the flt3 gene found in acute myeloid leukemia. Leukemia 10: 1911-1918, 1996.

5 Boyer SW, Schroeder AV, Smith-Berdan S and Forsberg EC: All hematopoietic cells develop from hematopoietic stem cells through Flk2/Flt3-positive progenitor cells. Cell Stem Cell 9: 6473, 2011.

6 Kiyoi H, Naoe T, Nakano Y, Yokota S, Minami S, Miyawaki S, Asou N, Kuriyama K, Jinnai I, Shimazaki C, Akiyama H, Saito $\mathrm{K}$, Oh H, Motoji T, Omoto E, Saito H, Ohno R and Ueda R: Prognostic implication of FLT3 and N-RAS gene mutations in acute myeloid leukemia. Blood 93: 3074-3080, 1999.

7 Papaemmanuil E, Gerstung M, Bullinger L, Gaidzik VI, Paschka P, Roberts ND, Potter NE, Heuser M, Thol F, Bolli N, Gundem G, Van Loo P, Martincorena I, Ganly P, Mudie L, McLaren S, O'Meara S, Raine K, Jones DR, Teague JW, Butler AP, Greaves MF, Ganser A, Dohner K, Schlenk RF, Dohner H and Campbell
PJ: Genomic Classification and Prognosis in Acute Myeloid Leukemia. N Engl J Med 374: 2209-2221, 2016.

8 Radomska HS, Alberich-Jorda M, Will B, Gonzalez D, Delwel $\mathrm{R}$ and Tenen DG: Targeting CDK1 promotes FLT3-activated acute myeloid leukemia differentiation through C/EBPalpha. J Clin Invest 122: 2955-2966, 2012.

9 Boutros R, Dozier C and Ducommun B: The when and wheres of CDC25 phosphatases. Curr Opin Cell Biol 18: 185-191, 2006.

10 Boutros R, Lobjois V and Ducommun B: CDC25 phosphatases in cancer cells: key players? Good targets? Nat Rev Cancer 7: 495-507, 2007.

11 Bertoli S, Boutzen H, David L, Larrue C, Vergez F, FernandezVidal A, Yuan L, Hospital MA, Tamburini J, Demur C, Delabesse E, Saland E, Sarry JE, Galcera MO, Mansat-De Mas V, Didier C, Dozier C, Recher C and Manenti S: CDC25A governs proliferation and differentiation of FLT3-ITD acute myeloid leukemia. Oncotarget 6: 38061-38078, 2015.

12 Brenner AK, Reikvam H, Lavecchia A and Bruserud O: Therapeutic targeting the cell division cycle 25 (CDC25) phosphatases in human acute myeloid leukemia - the possibility to target several kinases through inhibition of the various CDC25 isoforms. Molecules 19: 18414-18447, 2014.

13 Blomberg I and Hoffmann I: Ectopic expression of Cdc25A accelerates the $\mathrm{G}(1) / \mathrm{S}$ transition and leads to premature activation of cyclin E- and cyclin A-dependent kinases. Mol Cell Biol 19: 6183-6194, 1999.

14 Millar J, McGowan C, Jones R, Sadhu K, Bueno A, Richardson $\mathrm{H}$ and Russell P: cde25 M-phase inducer. Cold Spring Harb Symp Quant Biol 56: 577-584, 1991.

15 Yoshimi A, Toya T, Kawazu M, Ueno T, Tsukamoto A, Iizuka H, Nakagawa M, Nannya Y, Arai S, Harada H, Usuki K, Hayashi Y, Ito E, Kirito K, Nakajima H, Ichikawa M, Mano H and Kurokawa M: Recurrent CDC25C mutations drive malignant transformation in FPD/AML. Nat Commun 5: 4770, 2014.

16 Arreba-Tutusaus P, Mack TS, Bullinger L, Schnoder TM, Polanetzki A, Weinert S, Ballaschk A, Wang Z, Deshpande AJ, Armstrong SA, Dohner K, Fischer T and Heidel FH: Impact of FLT3-ITD location on sensitivity to TKI-therapy in vitro and in vivo. Leukemia 30: 1220-1225, 2016.

17 Heidel FH, Bullinger L, Feng Z, Wang Z, Neff TA, Stein L, Kalaitzidis D, Lane SW and Armstrong SA: Genetic and pharmacologic inhibition of beta-catenin targets imatinib-resistant leukemia stem cells in CML. Cell Stem Cell 10: 412-424, 2012.

18 Wang R, He G, Nelman-Gonzalez M, Ashorn CL, Gallick GE, Stukenberg PT, Kirschner MW and Kuang J: Regulation of Cdc25C by ERK-MAP kinases during the G2/M transition. Cell 128: 1119-1132, 2007.

19 Bonnet J, Coopman P, and Morris MC: Characterization of centrosomal localization and dynamics of Cdc25C phosphatase in mitosis. Cell Cycle 7: 1991-1998, 2008.

20 Strausfeld U, Fernandez A, Capony JP, Girard F, Lautredou N, Derancourt J, Labbe JC and Lamb NJ: Activation of p34cdc2 protein kinase by microinjection of human cdc $25 \mathrm{C}$ into mammalian cells. Requirement for prior phosphorylation of cdc25C by $\mathrm{p} 34 \mathrm{cdc} 2$ on sites phosphorylated at mitosis. J Biol Chem 269: 5989-6000, 1994.

21 Peng CY, Graves PR, Thoma RS, Wu Z, Shaw AS and PiwnicaWorms H: Mitotic and G2 checkpoint control: regulation of 143-3 protein binding by phosphorylation of $\mathrm{Cdc} 25 \mathrm{C}$ on serine216. Science 277: 1501-1505, 1997. 
22 Song Y, Li Z, Wang B, Xiao J, Wang X and Huang J: PhosphoCdc 25 correlates with activating $\mathrm{G}_{2} / \mathrm{M}$ checkpoint in mouse zygotes fertilized with hydrogen peroxide-treated mouse sperm. Mol Cell Biochem 396: 41-48, 2014.

23 Wang R, Jung SY, Wu CF, Qin J, Kobayashi R, Gallick GE and Kuang J: Direct roles of the signaling kinase RSK2 in Cdc25C activation during Xenopus oocyte maturation. Proc Natl Acad Sci USA 107: 19885-19890, 2010.

24 Toyoshima-Morimoto F, Taniguchi E and Nishida E: Plk1 promotes nuclear translocation of human $\mathrm{Cdc} 25 \mathrm{C}$ during prophase. EMBO Rep 3: 341-348, 2002.

25 Stubbs MC, Kim YM, Krivtsov AV, Wright RD, Feng Z, Agarwal J, Kung AL and Armstrong SA: MLL-AF9 and FLT3 cooperation in acute myelogenous leukemia: development of a model for rapid therapeutic assessment. Leukemia 22: 66-77, 2008.

26 Shlush LI and Mitchell A: AML evolution from preleukemia to leukemia and relapse. Best Pract Res Clin Haematol 28: 81-89, 2015.

27 Gohring G, Giagounidis A, Busche G, Hofmann W, Kreipe HH, Fenaux $\mathrm{P}$, Hellstrom-Lindberg $\mathrm{E}$ and Schlegelberger $\mathrm{B}$ : Cytogenetic follow-up by karyotyping and fluorescence in situ hybridization: implications for monitoring patients with myelodysplastic syndrome and deletion $5 \mathrm{q}$ treated with lenalidomide. Haematologica 96: 319-322, 2011.
28 Wei S, Chen X, Rocha K, Epling-Burnette PK, Djeu JY, Liu Q, Byrd J, Sokol L, Lawrence N, Pireddu R, Dewald G, Williams A, Maciejewski J and List A: A critical role for phosphatase haplodeficiency in the selective suppression of deletion 5q MDS by lenalidomide. Proc Natl Acad Sci USA 106: 12974-12979, 2009.

29 Caudill JS, Porcher JC and Steensma DP: Aberrant pre-mRNA splicing of a highly conserved cell cycle regulator, CDC25C, in myelodysplastic syndromes. Leuk Lymphoma 49: 989-993, 2008.

30 Skrdlant L, Stark JM and Lin RJ: Myelodysplasia-associated mutations in serine/arginine-rich splicing factor SRSF2 lead to alternative splicing of CDC25C. BMC Mol Biol 17: 18, 2016.

31 Chen MS, Hurov J, White LS, Woodford-Thomas $\mathrm{T}$ and Piwnica-Worms $\mathrm{H}$ : Absence of apparent phenotype in mice lacking Cdc25C protein phosphatase. Mol Cell Biol 21: 38533861,2001
Received September 11, 2016

Revised September 22, 2016

Accepted September 29, 2016 